\title{
Halo formation in three-dimensional bunches with various phase space distributions
}

\author{
A. V. Fedotov and R. L. Gluckstern \\ Physics Department, University of Maryland, College Park, Maryland 20742 \\ S. S. Kurennoy and R. D. Ryne \\ LANSCE Division, Los Alamos National Laboratory, Los Alamos, New Mexico 87545
}

(Received 14 July 1998; published 12 January 1999)

\begin{abstract}
A realistic treatment of halo formation must take into account $3 \mathrm{D}$ beam bunches and $6 \mathrm{D}$ phase space distributions. We recently constructed, analytically and numerically, a new class of self-consistent 6D phase space stationary distributions, which allowed us to study the halo development mechanism without being obscured by the effect of beam redistribution. In this paper we consider nonstationary distributions and study how the halo characteristics compare with those obtained using the stationary distribution. We then discuss the effect of redistribution on the halo development mechanism. In contrast to bunches with a large aspect ratio, we find that the effect of coupling between the $r$ and $z$ planes is especially important as the bunch shape becomes more spherical. [S1098-4402(98)00025-1]
\end{abstract}

PACS numbers: 41.85.-p, 29.27.Bd, 52.65.Rr

\section{INTRODUCTION}

The need for high current in a variety of new accelerator applications has focused a great deal of attention on understanding the phenomenon of halo formation in ion beams, which can cause excessive radioactivation of the accelerator. Starting in about 1991, a variety of twodimensional (2D) simulation studies [1-5] has led to the conclusion that halos are formed when a beam is mismatched to a focusing channel, exciting some sort of collective oscillation(s) of the beam which are in resonance with the nonlinear oscillation of individual ions.

Most of the simulation studies start with rms matched beams which are not stationary solutions of the Vlasov equation (see, for example, [6]). As a result, the initial beam undergoes a redistribution in phase space, obscuring the possible development of halos. Our effort has been devoted to populating a stationary distribution in phase space, in the expectation that the halo development mechanism can be studied without being obscured by the "relaxation" of the beam in phase space. We have particularly studied initial distributions which are stationary by virtue of being a function only of the Hamiltonian [7-9].

It is clear that a realistic treatment of halo formation must take into account 3D beam bunches and 6D phase space distributions. Barnard and Lund [10] performed numerical studies with a 3D beam bunch using the particle-core model, drawing attention to the existence and importance of a longitudinal halo for a spheroidal bunch. However, studies based on the particle-core model do not address the question of whether halo formation is influenced by the density redistribution which follows for a nonstationary beam, even if it is rms matched [6]. In fact, halo formation in 2D due to the redistribution process in rms matched beams was shown, for example, by Okamoto [11] and Jameson [2]. We therefore continued our effort to study the halo development mechanism in
3D beam bunches in the absence of the redistribution process [12]. Such an approach allowed us to study the fundamental mechanism of halo formation associated with the beam mismatch. To accomplish this we constructed a new class of stationary 6D phase space distributions for a spheroidal beam bunch [12]. We then explored the formation of longitudinal and transverse halos in 3D bunches in great detail [12].

Now that we have established the parameters which lead to halo formation in $3 \mathrm{D}$ beam bunches for the 6D self-consistent phase space distribution, we explore distributions which are not self-consistent to determine the extent to which the relatively rapid redistribution in the $6 \mathrm{D}$ phase space contributes to the formation of halos. This is the focus of the present paper.

\section{DIFFERENT 6D PHASE SPACE DISTRIBUTION}

We previously performed a detailed study for the azimuthally symmetric $6 \mathrm{D}$ phase space stationary distribution [12]:

$$
f(\boldsymbol{x}, \boldsymbol{p})= \begin{cases}N\left(H_{0}-H\right)^{-1 / 2}, & H<H_{0}, \\ 0, & H \geq H_{0},\end{cases}
$$

where

$$
H=k_{x} r^{2} / 2+k_{z} z^{2} / 2+e \Phi_{\mathrm{sc}}(\boldsymbol{x})+m v^{2} / 2
$$

and $H_{0}$ is a constant. Even though the ion motion may be relativistic in the laboratory frame, we transform to the Lorentz frame of the bunch, where all motion is nonrelativistic. The description of the effects in the laboratory frame can be obtained by an inverse Lorentz transformation should one wish to do so.

Here $\boldsymbol{p}=m \boldsymbol{v}, r^{2}=x^{2}+y^{2}$, and $k_{x}, k_{z}$ are the smoothed transverse and longitudinal restoring force gradients. The quantity $\Phi_{\mathrm{sc}}(\boldsymbol{x})$ is the electrostatic potential 
due to the space charge of the bunch. The distribution is normalized such that

$$
\int d \boldsymbol{x} \int d \boldsymbol{p} f(\boldsymbol{x}, \boldsymbol{p})=1
$$

The charge distribution corresponding to Eq. (1) is

$$
\begin{aligned}
\rho(\boldsymbol{x}) & =Q \int d \boldsymbol{p} f(\boldsymbol{x}, \boldsymbol{p}) \\
& =N Q m^{3} \int d \boldsymbol{v}\left[G(\boldsymbol{x})-\frac{m \boldsymbol{v}^{2}}{2}\right]^{-1 / 2},
\end{aligned}
$$

where

$$
G(\boldsymbol{x}) \equiv H_{0}-\frac{k_{x} r^{2}}{2}-\frac{k_{z} z^{2}}{2}-e \Phi_{\mathrm{sc}}(\boldsymbol{x}) .
$$

The integral in Eq. (4) extends over a sphere of radius $\boldsymbol{v}_{0}=[2 G(\boldsymbol{x}) / \mathrm{m}]^{1 / 2}$.

In this paper we compare particle simulations performed for the distribution given by Eq. (1) [12] with the nonstationary 6D Gaussian distribution

$$
\begin{aligned}
f(\boldsymbol{x}, \boldsymbol{v})= & N \exp \left[-\left(\boldsymbol{v}_{x}^{2}+\boldsymbol{v}_{y}^{2}+\boldsymbol{v}_{z}^{2}\right) /\left(2\left\langle\boldsymbol{v}_{x}^{2}\right\rangle\right)\right] \\
& \times \exp \left[-\left(x^{2}+y^{2}\right) /\left(2\left\langle x^{2}\right\rangle\right)-z^{2} /\left(2\left\langle z^{2}\right\rangle\right)\right]
\end{aligned}
$$

and the nonstationary $6 \mathrm{D}$ uniform distribution

$$
\begin{aligned}
f(\boldsymbol{x}, \boldsymbol{v})= & N \Theta\left(1-\frac{x^{2}}{a^{2}}-\frac{y^{2}}{a^{2}}-\frac{z^{2}}{c^{2}}\right) \\
& \times \Theta\left(1-\frac{v_{x}^{2}}{v_{1}^{2}}-\frac{v_{y}^{2}}{v_{1}^{2}}-\frac{v_{z}^{2}}{v_{3}^{2}}\right),
\end{aligned}
$$

where we consider an axisymmetric beam bunch by putting $a=b$ and $v_{1}=v_{2}$ with $a, c$ being the minor and major semiaxes of our spheroidal bunch, respectively. Both the Gaussian and uniform distributions are constructed in the rms matched sense.

To prevent possible future confusion, we remind the reader that, for an elongated 3D bunch $c / a>1$, the longitudinal tune depression $\eta_{z}$ is lower (more severe) than the transverse tune depression $\eta_{x}$ as long as the beam is equipartitioned before it is rms mismatched. (See [12] for a detailed discussion of this question.) Note that for our comparison with the 6D stationary distribution, which is equipartitioned, the rms values for the distributions in Eqs. (6) and (7) are chosen to be the same as those for the stationary distribution. In other words, we study beams described by Eqs. (6) and (7) which are initially equipartitioned.

\section{NUMERICAL SIMULATIONS}

\section{A. Implementation}

We have developed a 3D particle-in-cell (PIC) code to explore the distributions described above and to study halo formation. The single-particle equations of motion are integrated using a symplectic, split-operator technique
[13]. The space charge calculation uses area weighting ("cloud-in-cell") and implements open boundary conditions with the Hockney convolution algorithm [14]. The code runs on parallel computers and, in particular, the space charge calculation has been optimized for parallel platforms using the Ferrell-Bertschinger method [15]. We can easily perform a particle simulation with $1 \times 10^{7}$ particles, but, since no change was observed between results with $1 \times 10^{7}$ and $1 \times 10^{6}$, we employ $1 \times 10^{6}$ particles in our simulations to reduce execution time.

We initially populate the $6 \mathrm{D}$ phase space according to Eq. (1), (6), or (7), and then mismatch the $x, y, z$ coordinates by factors $\mu_{x}=\mu_{y}=1+\delta a / a, \mu_{z}=1+\delta c / c$ and the corresponding momenta by $1 / \mu_{x}=1 / \mu_{y}, 1 / \mu_{z}$.

As in our recent work [12], we will use an $r-p_{r}$ diagram to present the transverse halo and a $z-p_{z}$ diagram to present the longitudinal halo. Note that in $3 \mathrm{D}$ calculations $p_{r}$ is given by

$$
p_{r}^{2}=p_{x}^{2}+p_{y}^{2}-\frac{p_{\theta}^{2}}{r^{2}}=\frac{\left(x p_{x}+y p_{y}\right)^{2}}{r^{2}} .
$$

We will see that both the longitudinal $\left(z-p_{z}\right)$ and transverse $\left(r-p_{r}\right)$ phase spaces clearly show the typical "peanut" diagrams observed in 2D calculations.

Our exploration of halo effects involves only changes from initial parameters. The figures are therefore presented in relative units and can be scaled for particular applications as needed. For example, in the case of the stationary or uniform distribution we use $z / a, x / a$ with $a$ being the initial transverse radius of the bunch. For the Gaussian bunch, there is no sharp edge. Therefore, in this case the data are normalized to the transverse radius of the rms equivalent stationary distribution. The time evolution in our figures is presented in arbitrary time units. For an rms matched beam with $c / a=3$, one longitudinal breathing oscillation takes about 8 such units, while for $c / a=2$ it takes about 6 time units.

\section{B. Stability of the matched distribution}

The analytic theory for the self-consistent 2D matched Kapchinskij-Vladimirskij (KV) beam suggests that the beam becomes unstable for severe tune depression. Both numerical studies of the unstable modes and multiparticle simulations for the $2 \mathrm{D}$ breathing $\mathrm{KV}$ beam with zero mismatch confirmed that the beam is unstable for tune depressions below $\eta=0.4$ [7]. However, no halo was observed in the corresponding 2D simulations.

Similar studies for other 2D rms matched distributions which are not stationary solutions of the Vlasov equation showed the existence of a halo for severe tune depression and zero mismatch parameter [11]. The existence of a halo for such rms matched distributions was attributed to the unavoidable plasma oscillations generated by the initial density-redistribution process which is clearly shown in [11]. 


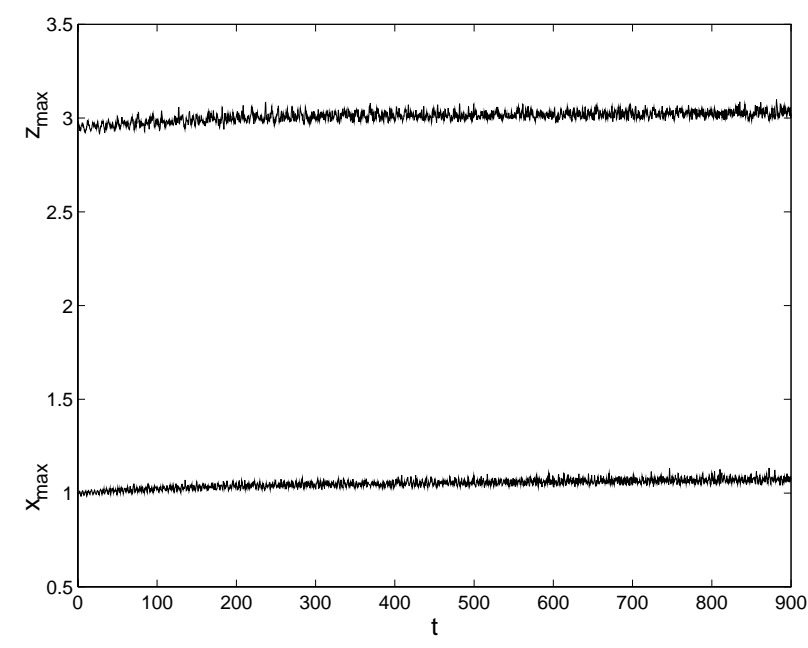

FIG. 1. Maximum $x$ and $z$ as a function of time for initially matched beam $\mu_{x}=\mu_{y}=\mu_{z}=1.0$ with 6D stationary distribution $\left(c / a=3, \eta_{x}=0.35, \eta_{z}=0.25\right)$.

In our recent $3 \mathrm{D}$ simulations [12] with the stationary distribution, no such redistribution occurred. In fact, an initially matched beam seems to be very stable, even for severe tune depressions. As an example, in Fig. 1 we plot the maximum $x$ and $z$ (scaled to the bunch radius, as described in Sec. III A) among the $1 \times 10^{6}$ particles in our run as a function of time for an initially matched beam, and in Fig. 2 we show the phase space diagram for $c / a=3, \eta_{x}=0.33, \eta_{z}=0.25$ with only 32768 particles plotted. We now perform similar 3D particle simulations for the nonstationary Gaussian and uniform distributions.

For the Gaussian distribution, one can see the strong redistribution process which occurs very quickly in both the transverse and longitudinal planes. In contrast to the 2D simulations [11], this redistribution process happens for both modest and severe space charge. In Fig. 3 we plot the maximum $x$ and $z$ among the $1 \times 10^{6}$ particles in our run for severe $\left(\eta_{z}=0.27, \eta_{x}=0.38\right)$ and modest $\left(\eta_{z}=0.65, \eta_{x}=0.75\right)$ tune depressions, respectively. Figure 4 shows the phase space $z-p_{z}$ diagram for $\eta_{z}=0.27, \eta_{x}=0.38$ without and with a low-density cut which enables us to observe the halo structure clearly. The low-density cut technique allows us to get better visual resolution in the halo region. In this procedure we choose a threshold phase space density just above that in the halo and plot all halo particles. In the high-density region, we plot only those particles in regions below the threshold density. From now on the longitudinal phase space diagram will be presented using this low-density cut technique (with only 20000 particles plotted) unless it is stated otherwise. Figure 5 shows the development of halo with zero initial mismatch (mismatch parameter $\mu_{x}=$ $\left.\mu_{y}=\mu_{z}=1.0\right)$ for a modest tune depression $\left(\eta_{z}=\right.$ $\left.0.65, \eta_{x}=0.75\right)$ at different time steps. At $t=50$ the beam performed only a few longitudinal oscillations, which shows how quickly the halo forms due to the redistribution process for a Gaussian distribution.

Similarly, one can see the redistribution process for the uniform distribution. One again finds halo formation for both modest and severe space charge, as can be seen in Figs. 6 and 7.

Thus, we have shown that an rms matched 3D beam can produce transverse and/or longitudinal halos for a wide range of space charge intensity even when it is initially perfectly matched. Of course, from a practical point of view such halos are not important because the halo extent is very small for the mismatch factor $\mu=1.0$ (the detailed study of the halo extent on a mismatch factor was presented in [12]).

The redistribution process in a nonstationary equipartitioned beam without initial rms mismatch causes the core to perform oscillations with a rapid increase of the maximum particle amplitude. This growth of the maximum beam size is quickly saturated. For example, for the uniform distribution with $c / a=3$ and tune depressions $\eta_{z}=0.35, \eta_{x}=0.49$, the increase of the maximum beam size is of the order of $10 \%$ and $30 \%$ of the initial beam size in the longitudinal and transverse directions, respectively (the numbers depend on the specific distribution and tune depression). This maximum amplitude is approximately the same as the maximum amplitude reached
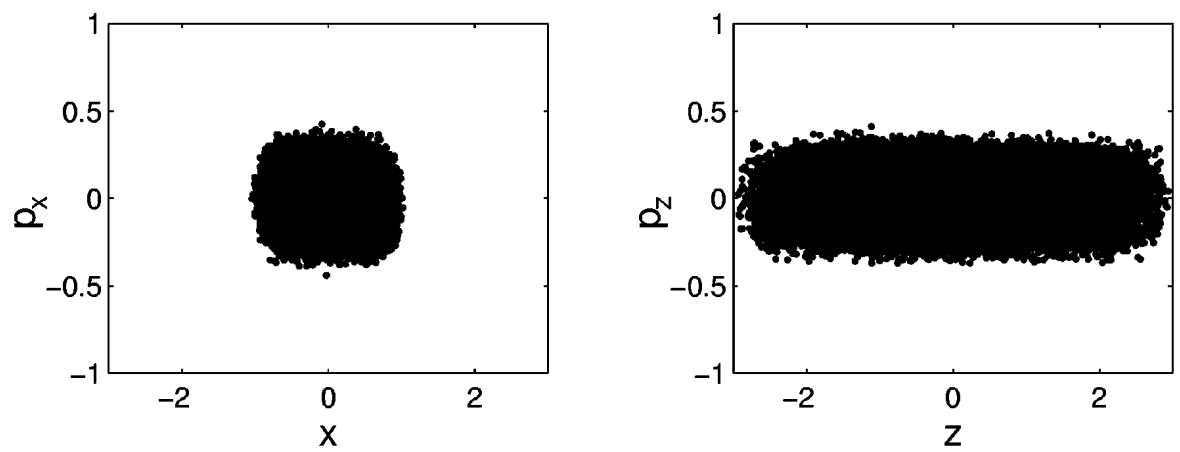

FIG. 2. Longitudinal phase space diagram at $t=900$ of initially matched beam $\mu_{x}=\mu_{y}=\mu_{z}=1.0$ for the $6 \mathrm{D}$ stationary distribution with 32768 particles plotted $\left(c / a=3, \eta_{x}=0.35, \eta_{z}=0.25\right)$. 
(a)

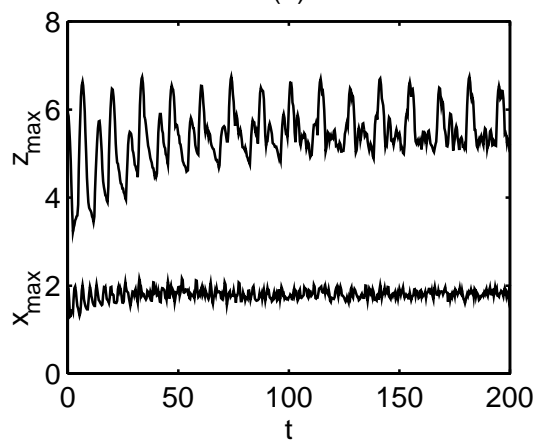

(b)

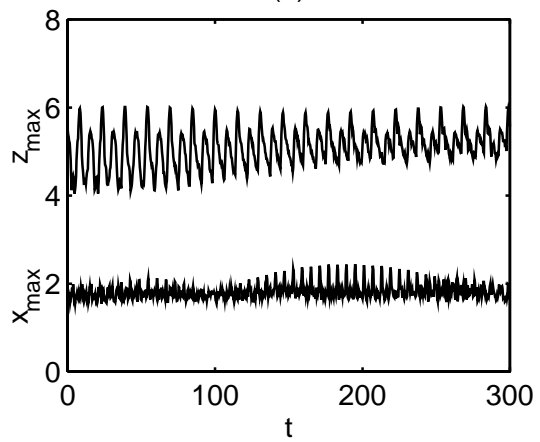

FIG. 3. Maximum $x$ and $z$ as a function of time for initially matched beam $\mu_{x}=\mu_{y}=\mu_{z}=1.0$ with 6D Gaussian distribution $(c / a=3)$ : (a) $\eta_{x}=0.38, \eta_{z}=0.27$, (b) $\eta_{x}=0.75, \eta_{z}=0.65$.

after significantly longer time by a beam having the stationary distribution, which is initially rms mismatched by only a few percent in the transverse or longitudinal plane, for the transverse or longitudinal halo, respectively. Therefore, the redistribution process produces the same maximum beam size as a few percent rms mismatch (in the corresponding plane) of the stationary beam.

We should also note that there is no straightforward description of the redistribution process in terms of some equivalent rms mismatch of the stationary beam. For example, for the stationary distribution, the halo extent is almost independent of the tune depression for very small mismatches, while the redistribution process in nonstationary beams clearly depends on the tune depression. Also, the time scales for the two effects are completely different. The redistribution process is extremely fast compared to the relatively slow halo formation due to the rms mismatch of stationary beams (see, for example, [6]). In addition, the other characteristics of the halo, such as the onset and rate of halo formation, strongly depend on tune depression for the stationary distribution [12], in contrast with the redistribution process. In Figs. 8 and 9, we show the trends of the maximum particle amplitude as a function of tune depression for rms matched uniform and Gaussian distributions, respectively. The maximum amplitude in transverse and longitudinal directions is plotted versus transverse or longitudinal tune depression, respectively.

The process of redistribution towards the equilibrium state in 6D phase space is related to the free energy and emittance growth in nonstationary beams. The studies of this process for continuous beam were summarized in [6], and were extended by Hofmann and Struckmeier to bunched beams [16]. The mechanism of the redistribution process should be studied in greater detail, but it is beyond the scope of the present paper. For the present purposes, we use the words "small equivalent mismatch" mainly to indicate that the effect of the redistribution process on halo formation is small rather than trying to make direct analogy between the density redistribution and the rms mismatch.

It is worth noting that the redistribution process by itself in the nonstationary distributions, without initial rms mismatch, does not lead to significant emittance growth. This can be seen in Fig. 10 for the Gaussian and uniform distributions with relatively strong tune depressions $\eta_{z}=0.27, \eta_{x}=0.38$. Such behavior is expected, since emittance growth is a strong function of the mismatch (see, for example, [6]). (a)

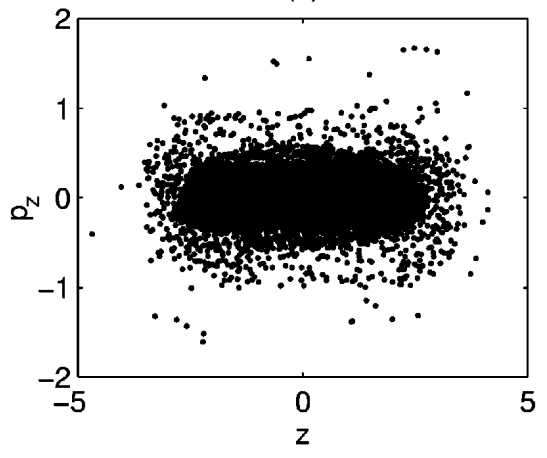

(b)

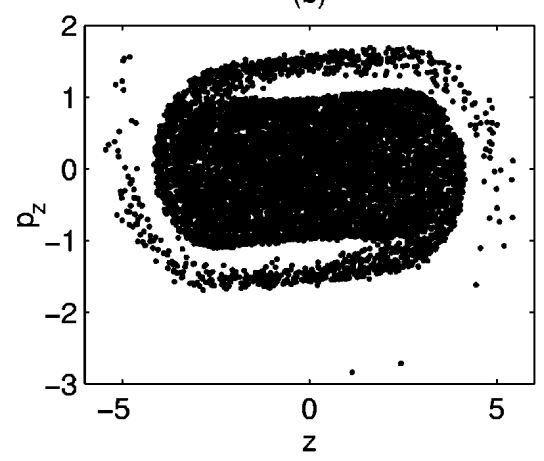

FIG. 4. Longitudinal phase space diagram at $t=50$ of initially matched beam $\mu_{x}=\mu_{y}=\mu_{z}=1.0$ with $6 \mathrm{D}$ Gaussian distribution $\left(c / a=3, \eta_{x}=0.38, \eta_{z}=0.27\right)$ : (a) without low-density cut (with 32768 particles plotted) and (b) with low-density cut (with 20000 particles plotted). 
(a)

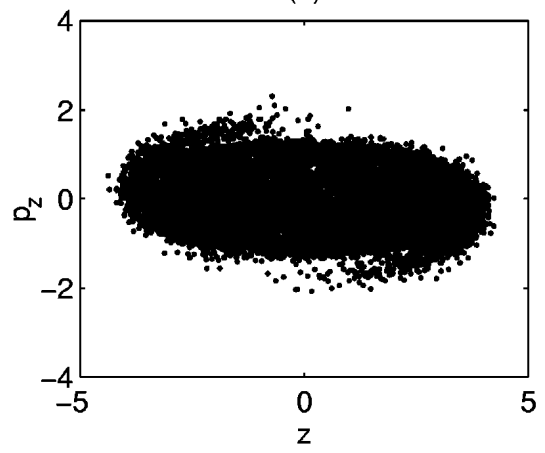

(c)

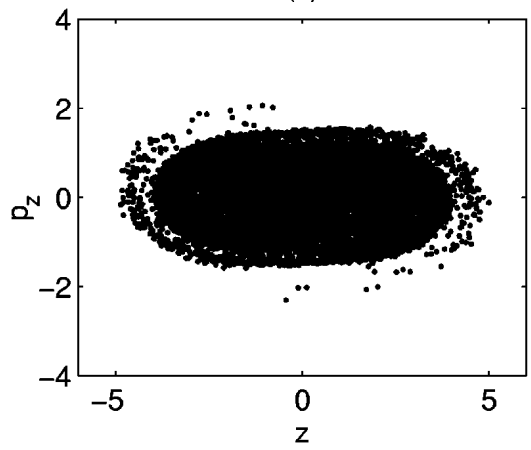

(b)

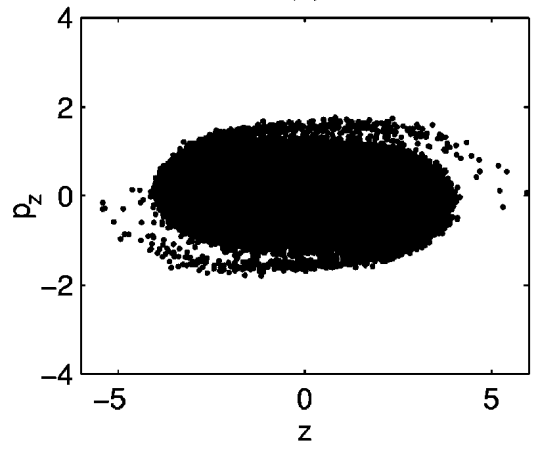

(d)

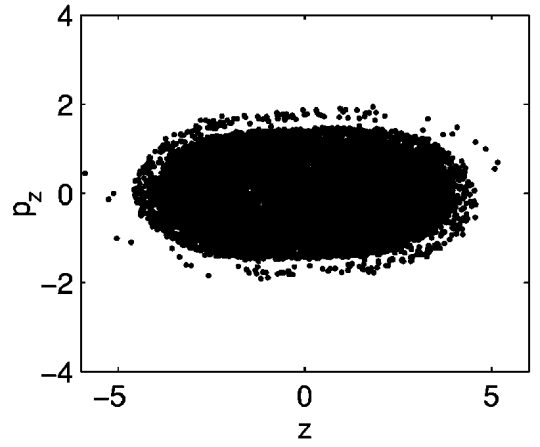

FIG. 5. Longitudinal phase space diagram at different time steps of initially matched beam $\mu_{x}=\mu_{y}=\mu_{z}=1.0$ with $6 \mathrm{D}$ Gaussian distribution $\left(c / a=3, \eta_{x}=0.75, \eta_{z}=0.65\right.$ ): (a) $t=50$, (b) $t=100$, (c) $t=150$, and (d) $t=200$.

(a)

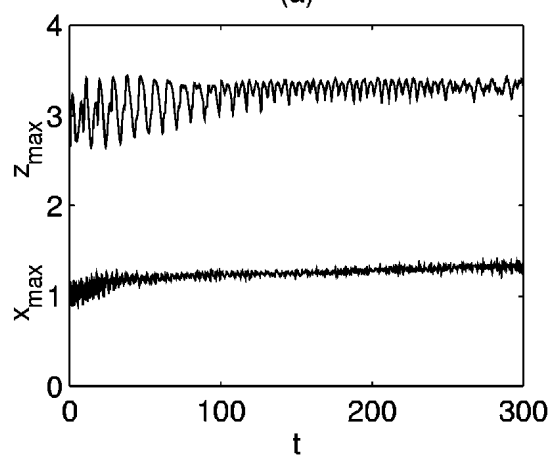

(b)

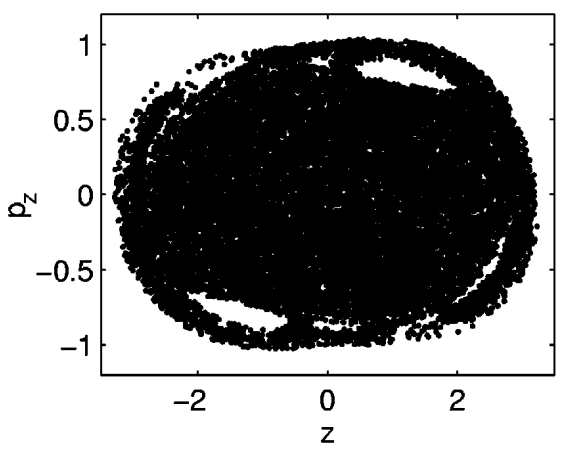

FIG. 6. 6D uniform distribution with initial zero mismatch $\left(c / a=3, \eta_{x}=0.75, \eta_{z}=0.65\right)$ : (a) maximum $x$ and $z$ and (b) $z-p_{z}$ diagram with the low-density cut.

(a)

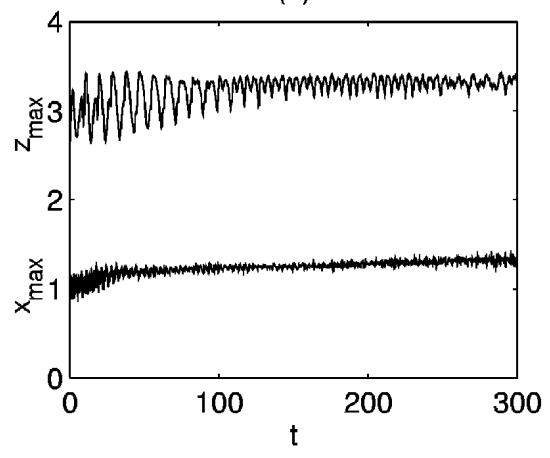

(b)

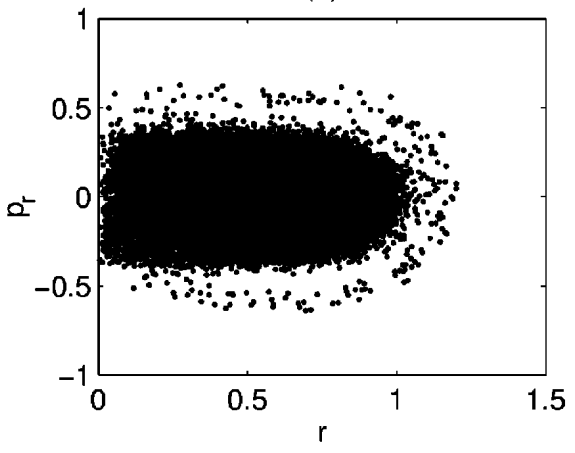

FIG. 7. 6D uniform distribution with initial zero mismatch $\left(c / a=3, \eta_{x}=0.38, \eta_{z}=0.27\right)$ : (a) maximum $x$ and $z$ and (b) $r$ - $p_{r}$ diagram at $t=300$ (with 25000 particles plotted). 


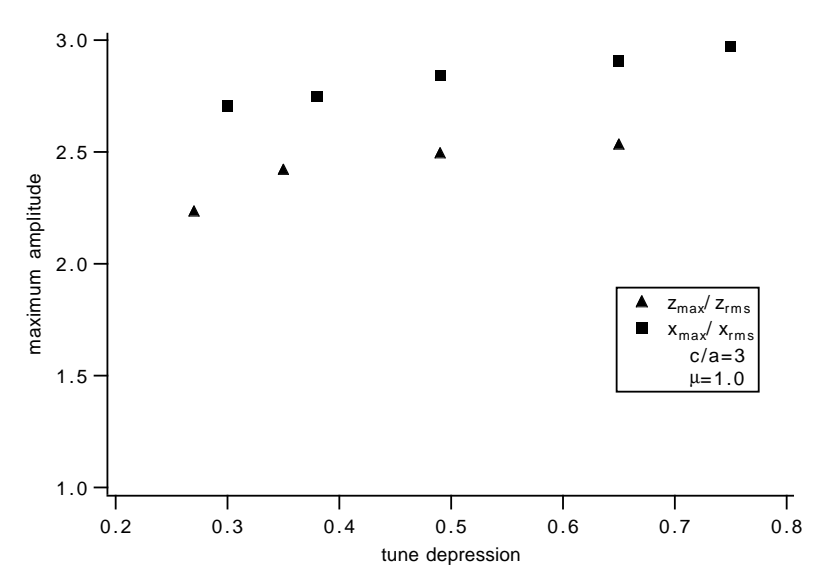

FIG. 8. The maximum particle amplitude in transverse and longitudinal directions vs transverse or longitudinal tune depression for an rms matched uniform distribution.

\section{Initially mismatched beam}

Numerical 3D simulations with the initially mismatched nonstationary distributions described above confirm all the characteristics of halos observed for the stationary distribution [12]. The main difference is that for a nonstationary distribution the halo extent is larger (especially for the Gaussian) than the halo extent of the stationary distribution with the same initial mismatch parameters. As an example, in Figs. 11-13 we show the maximum $x, z$, emittance growth, $z-p_{z}$ diagram without the low-density cut, and $r-p_{r}$ diagram (with angular momentum $\left|L_{z}\right|<$ 0.1 to make the peanut diagram relatively clear) with initial $\mu_{x}=\mu_{y}=\mu_{z}=1.5$ for the Gaussian, uniform and stationary distributions, respectively. Once again, we explore effects which involve only changes from initial parameters. Thus, momentum in phase space diagrams, maximum $x$ and $z$, and emittance are presented in rela-

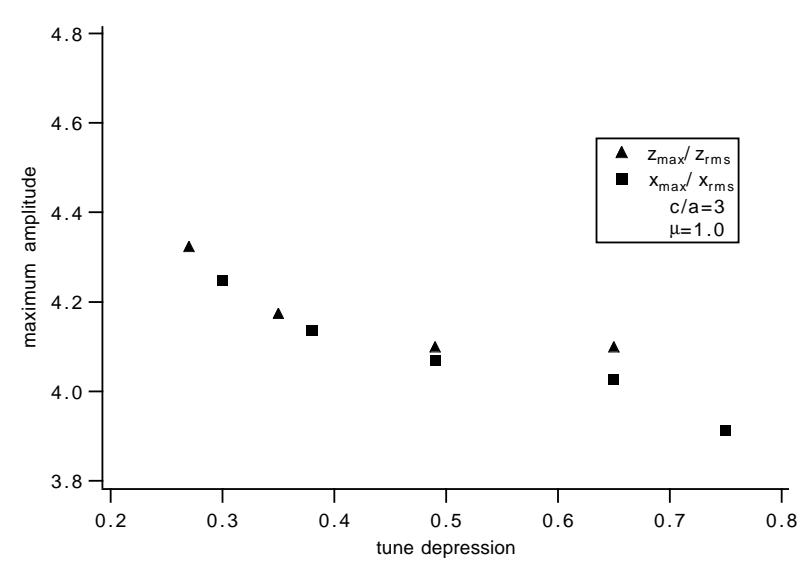

FIG. 9. The maximum particle amplitude in transverse and longitudinal directions vs transverse or longitudinal tune depression for an rms matched Gaussian distribution. tive units and can be scaled for particular applications as needed (see Sec. III A).

One can see that the 6D stationary distribution constructed in [12] gives a picture of halo development almost identical to the uniform nonstationary distribution except for a slight difference in the halo extent. Despite the fact that the Gaussian distribution does not precisely describe a "real" beam in the space charge limit in a linac, it gives an approximate upper limit on the halo extent, which can be very useful in practical applications.

It was shown [12] that for a 3D bunch one generates both transverse (breathing) and longitudinal modes by introducing arbitrary mismatch in any direction. In fact, a primarily longitudinal or transverse mode can develop only if the relation between the transverse and longitudinal mismatches is satisfied [12]. Otherwise, one always has a combination of both modes and therefore the possibility that both longitudinal and transverse halos develop. A systematic study for bunches of different shape $(c / a)$ and mismatch factor $\mu$ (with simultaneous mismatch in all planes) was presented recently [12]. In the following, we present some examples of the mismatch in either the transverse or longitudinal plane only. To compare our results with those available for a transverse halo [17], we show in Fig. 14 the dependence of the transverse halo extent on the mismatch for fixed space charge, with tune depressions $\eta_{z}=0.39, \eta_{x}=0.53$. Our plot, obtained using the $6 \mathrm{D}$ stationary distribution, is in very good agreement with the results presented by Wangler et al. [17]. The main difference is the behavior near $\mu=1.0$ which shows some sort of a threshold for halo formation in the transverse plane for beams with a stationary distribution. The existence of a similar threshold is not so obvious for the longitudinal halo, as can be seen in Fig. 15 for tune depressions $\eta_{z}=0.49, \eta_{x}=0.65$. In fact, numerical runs for more severe tune depressions $\eta_{z}=0.35, \eta_{x}=0.49$ did not show any sign of the existence of a threshold for the longitudinal halo at all. Also, one should distinguish the situation discussed above from the case with a simultaneous mismatch in all planes. For the latter case, the longitudinal halo can be produced more easily because of the coupling. It is worth noting that the extent of the longitudinal halo is smaller than that of the transverse halo, as can be seen from Figs. 14 and 15.

The existence of a threshold for halo formation in the transverse plane, which was observed in $2 \mathrm{D}$ simulations and is also seen in our 3D particle simulations, seems to be a feature observed only for self-consistent stationary distributions. For example, Fig. 16 for the 6D uniform nonstationary distribution has no obvious transverse threshold behavior. In fact, as we showed above, in a nonstationary beam a halo can form even for a zero initial mismatch.

For practical application, we also note that there is no significant difference in the halo extent between the situation where the mismatch is in a single plane and 
(a)

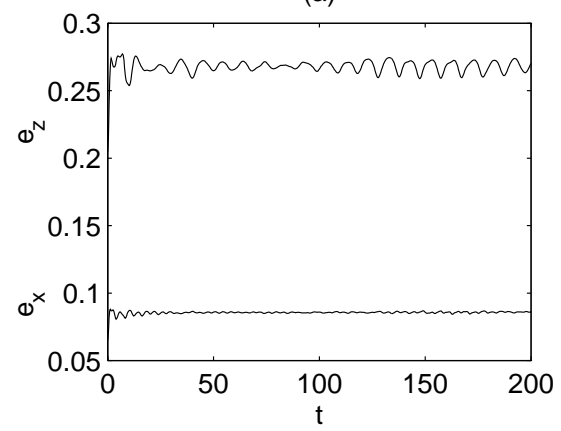

(b)

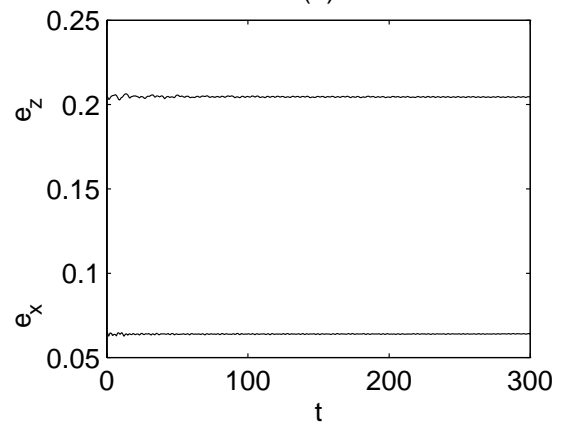

FIG. 10. Emittance growth due to the redistribution process for initially matched beam $\left(c / a=3, \eta_{x}=0.38, \eta_{z}=0.27\right)$ : (a) Gaussian distribution and (b) uniform distribution.

the one where the simultaneous mismatch is in both planes, but there is a substantial difference in the halo intensity. For example, consider a beam bunch with a 6D stationary distribution and parameters $c / a=3, \eta_{z}=$ $0.49, \eta_{x}=0.65$ initially mismatched by $30 \%$ in only the longitudinal direction $\left(\mu_{z}=1.3, \mu_{x}=\mu_{y}=1.0\right)$. When the same bunch is instead initially mismatched by $30 \%$ in all directions $\left(\mu_{z}=\mu_{x}=\mu_{y}=1.3\right)$ the extent of the longitudinal halo after its rough saturation (more than 100 longitudinal breathing oscillations) increases by only a few percent, while the number of the particles in the longitudinal halo increases greatly (from 0.06 to about $1 \%$ of particles in the halo).

(a)

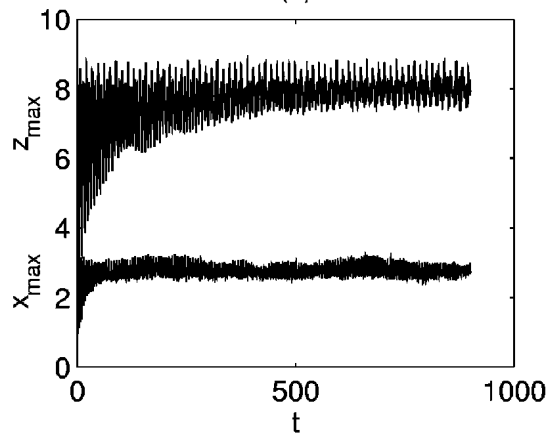

(c)

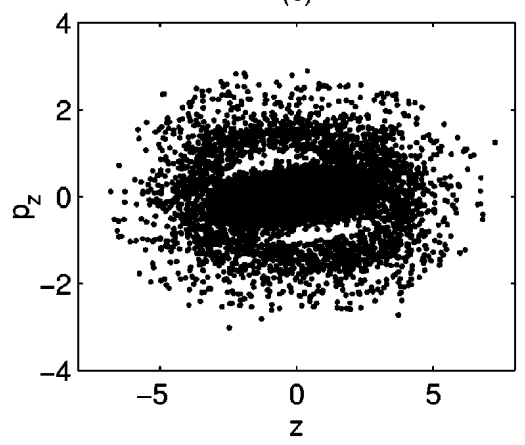

\section{Coupling effects}

In performing 3D simulations we encounter halo formation in a beam bunch, where we clearly see coupling between the longitudinal and transverse motion. It was already noted [12] that due to the coupling between $r$ and $z$, a transverse or longitudinal halo is observed even for a very small mismatch (less than $10 \%$ ) as long as there is a significant mismatch in the other plane. Further numerical investigation of this question showed that the effect of coupling becomes extremely important for nearly spherical bunches $(c / a \leq 2)$, which is typical of the parameter range of interest for the accelerator production of tritium

(b)

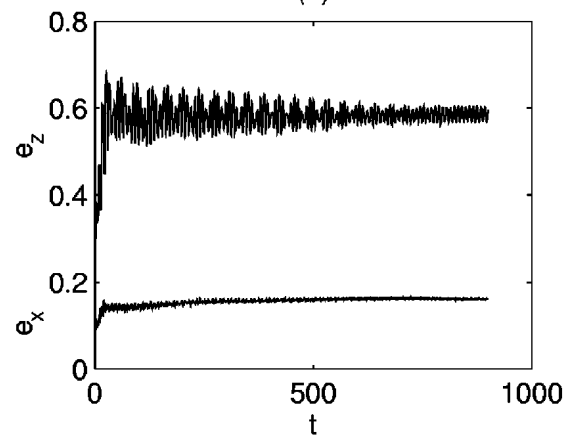

(d)

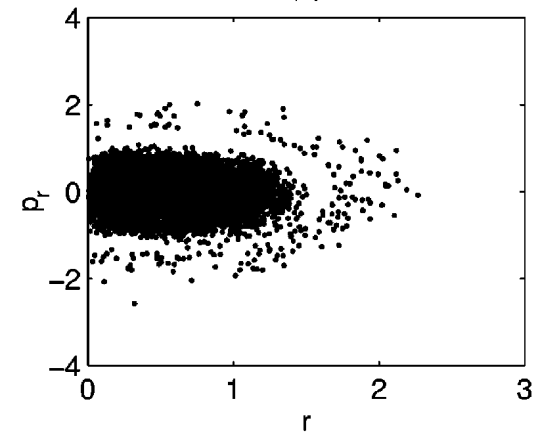

FIG. 11. 6D Gaussian distribution $\mu_{x}=\mu_{y}=\mu_{z}=1.5\left(c / a=3, \eta_{x}=0.53, \eta_{z}=0.39\right.$ ): (a) maximum $x$ and $z$, (b) emittance growth, (c) $z-p_{z}$ diagram at $t=900$ (with 32768 particles plotted), and (d) $r$ - $p_{r}$ diagram at $t=900$ for particles with the angular momentum $\left|L_{z}\right|<0.1$ (with 25000 particles plotted). 
(a)

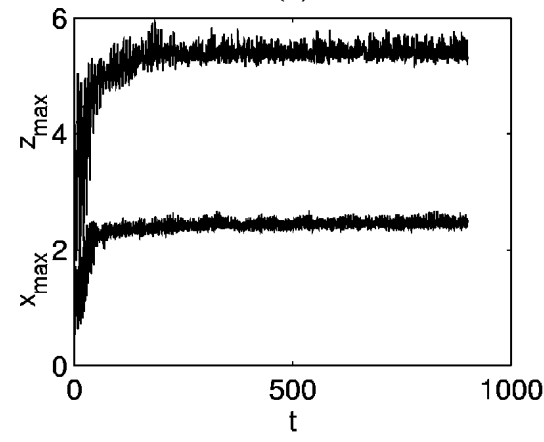

(c)

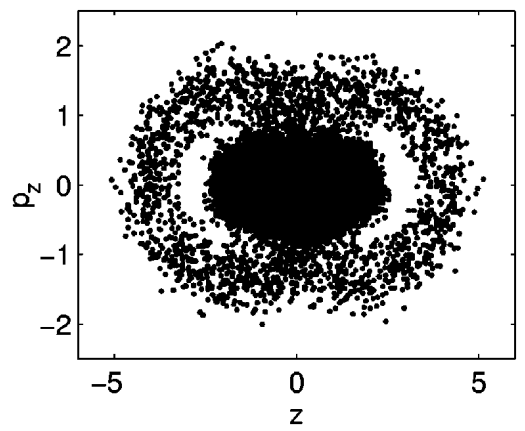

(b)

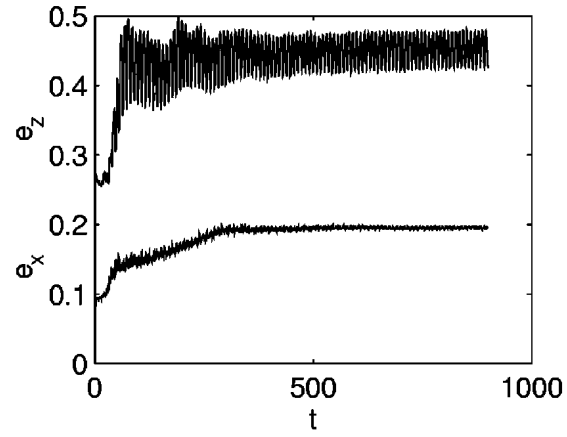

(d)

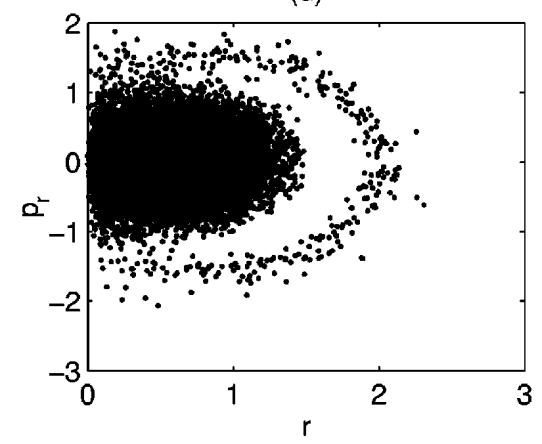

FIG. 12. 6D uniform distribution $\mu_{x}=\mu_{y}=\mu_{z}=1.5\left(c / a=3, \eta_{x}=0.53, \eta_{z}=0.39\right)$ : (a) maximum $x$ and $z$, (b) emittance growth, (c) $z-p_{z}$ diagram at $t=900$ (with 32768 particles plotted), and (d) $r-p_{r}$ diagram at $t=900$ for particles with the angular momentum $\left|L_{z}\right|<0.1$ (with 25000 particles plotted).

(a)

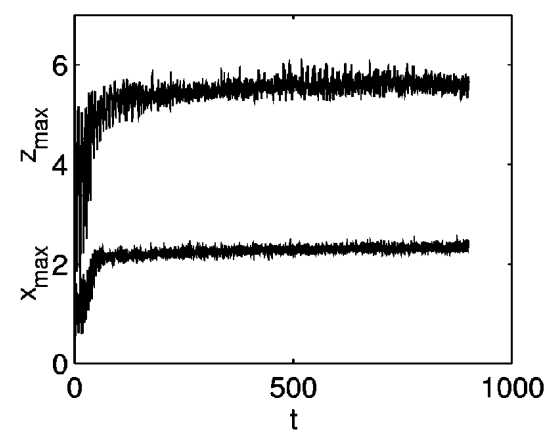

(c)

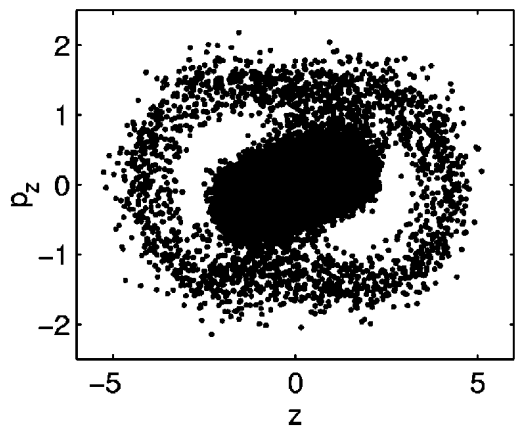

(b)

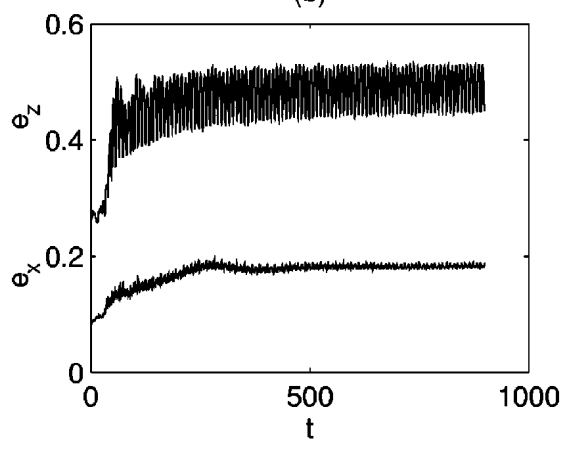

(d)

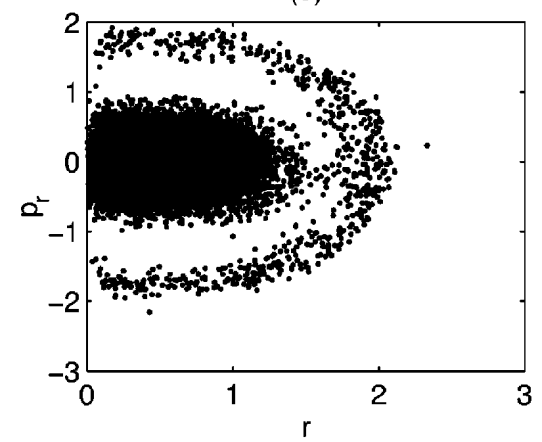

FIG. 13. 6D stationary distribution $\mu_{x}=\mu_{y}=\mu_{z}=1.5\left(c / a=3, \eta_{x}=0.53, \eta_{z}=0.39\right)$ : (a) maximum $x$ and $z$, (b) emittance growth, (c) $z-p_{z}$ diagram at $t=900$ (with 32768 particles plotted), and (d) $r-p_{r}$ diagram at $t=900$ for particles with the angular momentum $\left|L_{z}\right|<0.1$ (with 2500 particles plotted). 


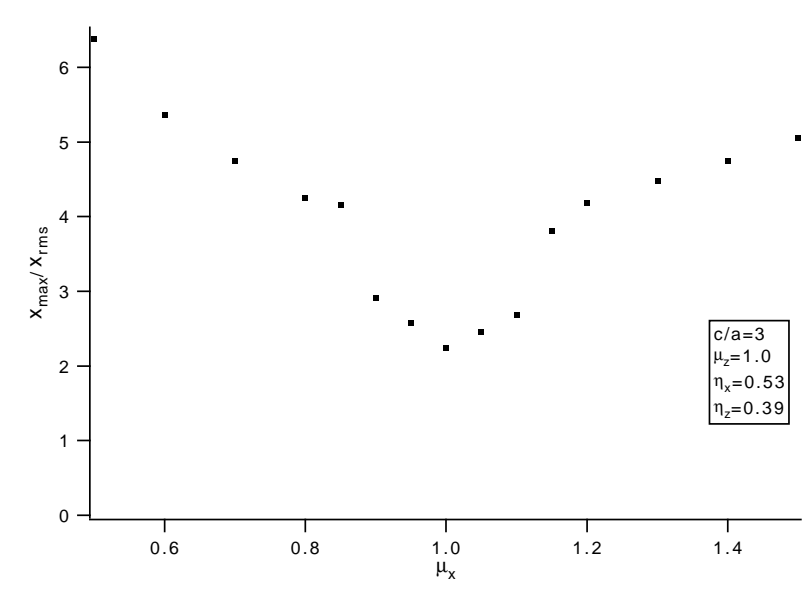

FIG. 14. Extent of the transverse halo for the 6D stationary distribution with zero longitudinal mismatch $\mu_{z}=1.0(\mathrm{c} / \mathrm{a}=$ $3, \eta_{x}=0.53, \eta_{z}=0.39$ ).

(APT) design [18]. For example, for the short bunch with $c / a=2$, with only a longitudinal initial mismatch $\left(\mu_{z}=1.5, \mu_{x}=\mu_{y}=1.0\right.$ ), one finds particles at large amplitude in both the longitudinal and transverse directions, as can be seen in Fig. 17 for the 6D stationary distribution. Of course, the intensity of particles in the transverse halo is much smaller than it is when there is, additionally, a transverse initial mismatch. (In our example in Fig. 17, we have $0.05 \%$ of the particles in the transverse halo with zero transverse mismatch compared with several percent in the longitudinal halo.) A similar effect due to coupling can be seen for the nonstationary distributions, as shown in Figs. 18 and 19. In fact, this effect appears to be stronger in nonstationary distributions. Thus, because of the coupling, a significant mismatch in any plane can be dangerous and should be treated carefully.

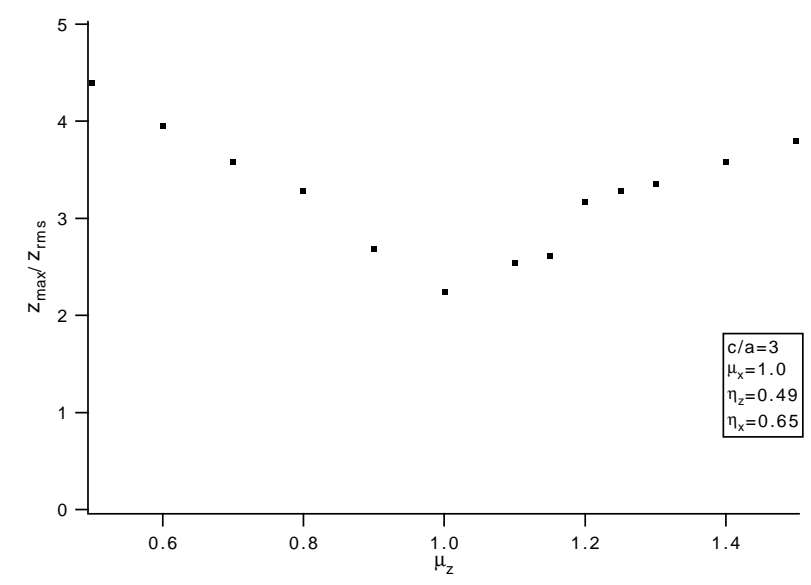

FIG. 15. Extent of the longitudinal halo for the 6D stationary distribution with zero transverse mismatch $\mu_{x}=\mu_{y}=1.0$ $\left(c / a=3, \eta_{x}=0.65, \eta_{z}=0.49\right)$.

\section{DISCUSSION}

The most important examples of a nonstationary beam are a beam with an rms mismatch, a nonstationary distribution function (the distribution function is not a solution of the stationary Vlasov equation), and a misaligned beam. The theory describing each of these dominant effects has been established, and a detailed description with references to the literature on this subject can be found in [6]. If the beam is initially nonstationary, it has a higher average energy per particle than the stationary beam. The energy difference represents the free energy that can be "thermalized" by nonlinear spacecharge forces, collisions, or instabilities. As a result of such a process, the emittance increases as the beam relaxes toward its final quasistationary state.

Among the three examples of nonstationary beam listed above, the rms mismatch is the largest contribution to the emittance growth [6]. Emittance growth is a strong function of the initial mismatch parameter. In order to study this effect in 3D, we recently constructed a new class of 6D phase space stationary distributions and explored its effect on halo formation in great detail [12]. However, real beams have a nonstationary density profile. In the previous section we showed that the redistribution process for beams with nonstationary distributions leads to oscillations of the beam radius about an average value, which is "equivalent" to introducing a small initial mismatch for a stationary distribution (see Sec. III B). Such a mismatch is largest for the 6D Gaussian distribution. Although this equivalent mismatch is relatively small, it can lead to the development of a halo of small extent because it is enhanced due to the coupling between the transverse and longitudinal motion in short $3 \mathrm{D}$ beam bunches. As a consequence of this effect, there is no threshold on halo formation due to the initial rms mismatch in real beams, as shown in Sec. III. The formation of a halo in a beam with a nonstationary distribution which is initially rms

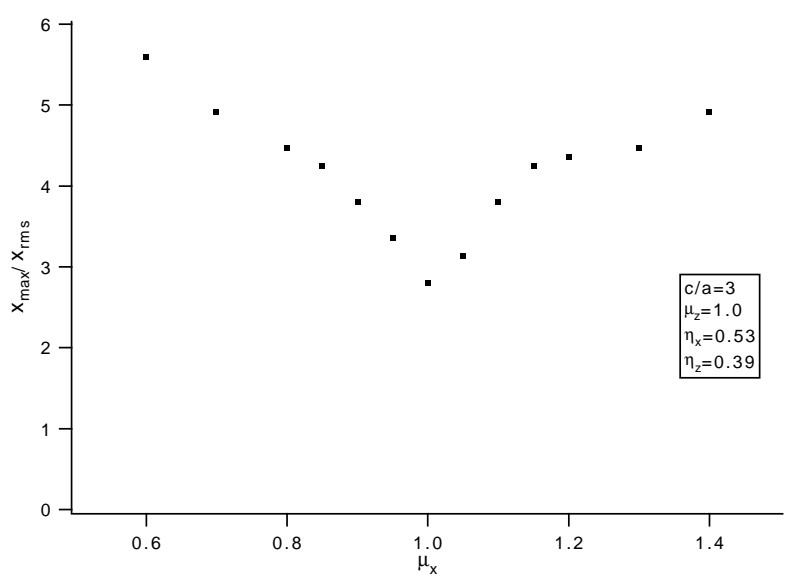

FIG. 16. Extent of the transverse halo for the 6D uniform distribution with zero longitudinal mismatch $\mu_{z}=1.0(\mathrm{c} / \mathrm{a}=$ $3, \eta_{x}=0.53, \eta_{z}=0.39$ ). 
(a)

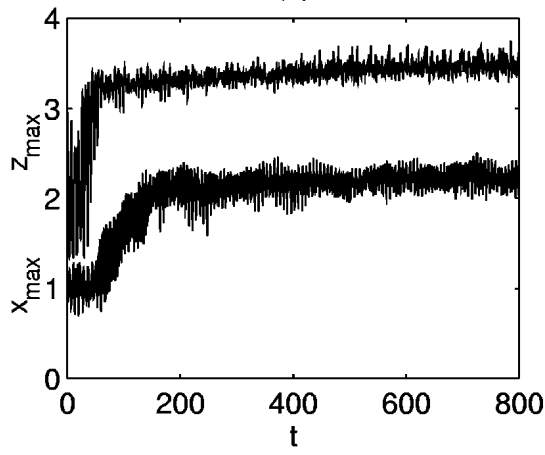

(b)

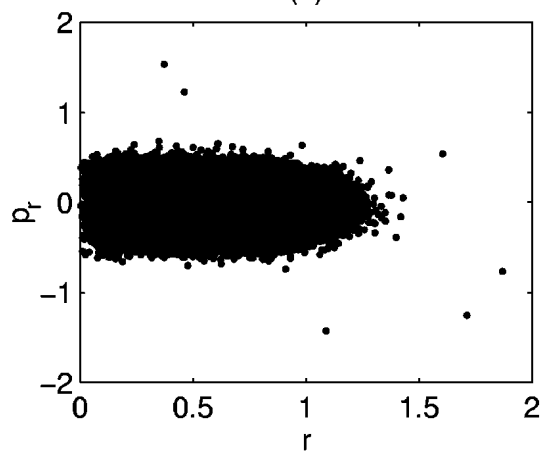

FIG. 17. Coupling effect for the 6D stationary distribution with zero transverse mismatch $\mu_{x}=\mu_{y}=1.0, \mu_{z}=1.5(c / a=$ 2, $\eta_{x}=0.55, \eta_{z}=0.45$ ): (a) maximum $x$ and $z$ and (b) $r$ - $p_{r}$ diagram at $t=800$ for particles with the angular momentum $\left|L_{z}\right|<0.1$ (with 25000 particles plotted).

(a)

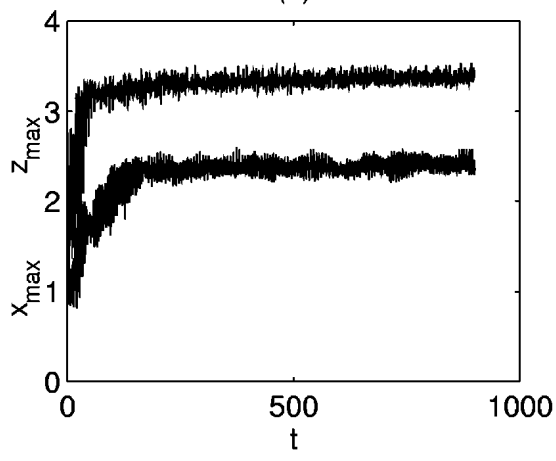

(b)

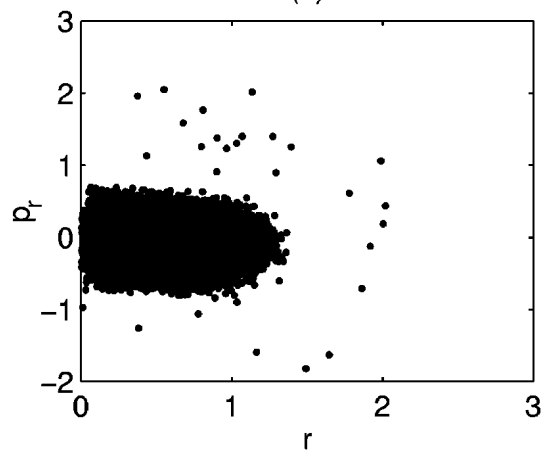

FIG. 18. Coupling effect for the 6D uniform distribution with zero transverse mismatch $\mu_{x}=\mu_{y}=1.0, \mu_{z}=1.5(c / a=$ 2, $\eta_{x}=0.55, \eta_{z}=0.45$ ): (a) maximum $x$ and $z$ and (b) $r$ - $p_{r}$ diagram at $t=900$ for particles with the angular momentum $\left|L_{z}\right|<0.1$ (with 25000 particles plotted).

(a)

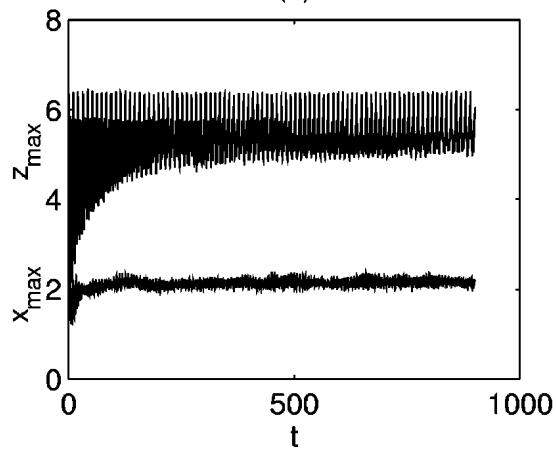

(b)

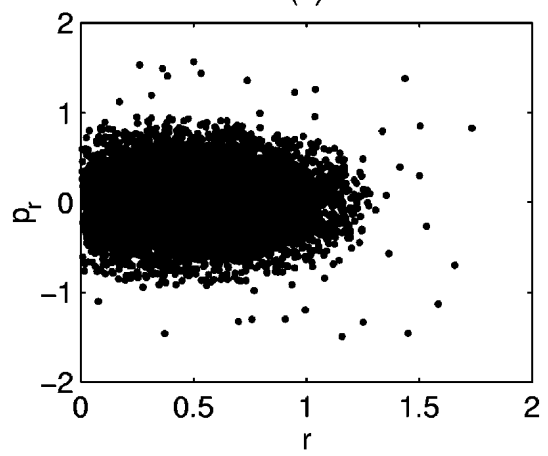

FIG. 19. Coupling effect for the 6D Gaussian distribution with zero transverse mismatch $\mu_{x}=\mu_{y}=1.0, \mu_{z}=1.5(c / a=$ $2, \eta_{x}=0.55, \eta_{z}=0.45$ ): (a) maximum $x$ and $z$ and (b) $r-p_{r}$ diagram at $t=900$ for particles with the angular momentum $\left|L_{z}\right|<0.1$ (with 25000 particles plotted).

mismatched of course has contributions from both the rms mismatch and the redistribution process.

Below, we briefly summarize the effect of the rms mismatch and redistribution process on equipartitioning. In our numerical simulations we start with a beam which is initially equipartitioned. For the 6D stationary distribution with initial zero rms mismatch, one can see that equipartitioning is preserved, as shown in Fig. 20. However, if the initial mismatch is not zero, one sees that the relaxation process proceeds toward a more stationary final state. The final transverse and longitudinal thermal states are not exactly the same, with a small anisotropy 


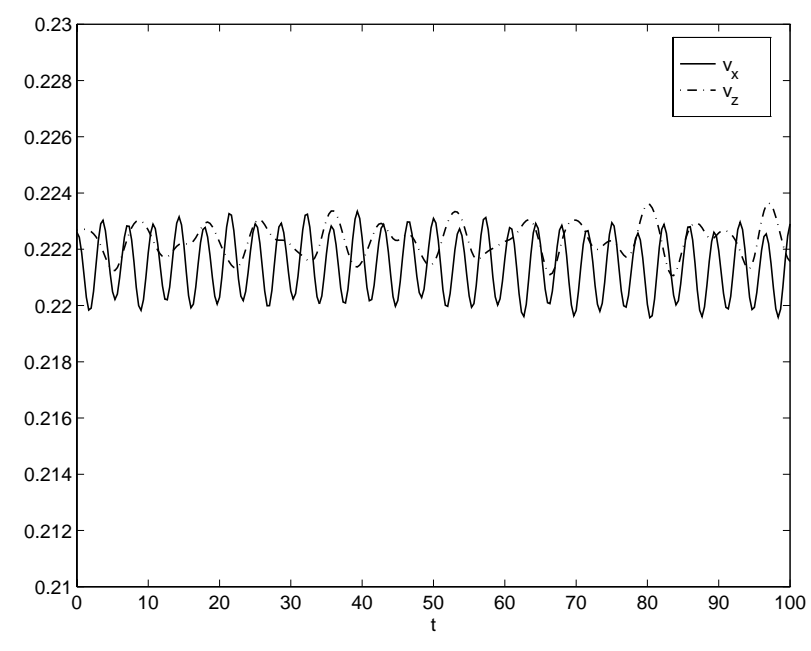

FIG. 20. rms velocities (in arbitrary units) for initially matched 6D stationary distribution $\left(c / a=3, \mu_{x}=\mu_{y}=\right.$ $\left.\mu_{z}=1.0, \eta_{x}=0.53, \eta_{z}=0.39\right)$.

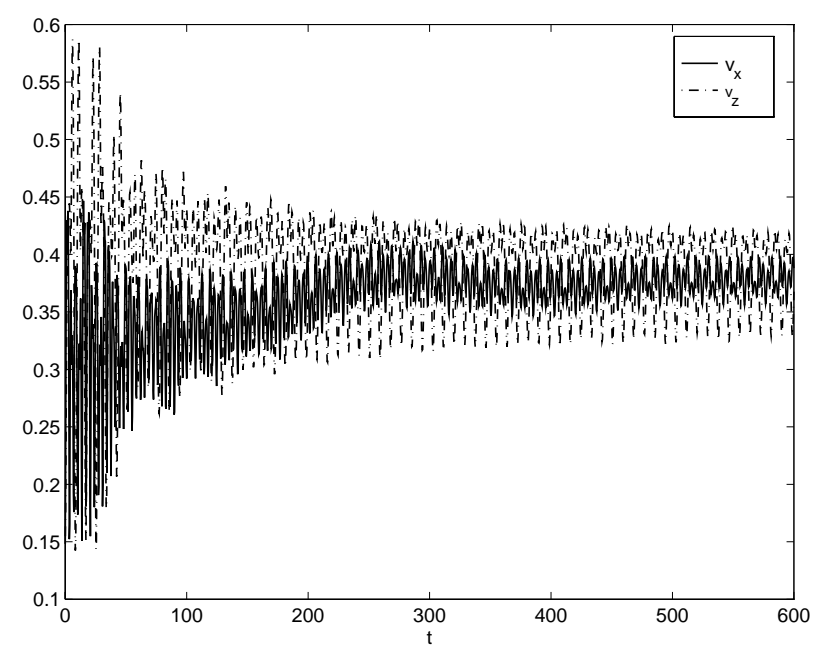

FIG. 21. rms velocities (in arbitrary units) for the 6D stationary distribution with strong initial mismatch $\left(c / a=3, \mu_{x}=\right.$ $\mu_{y}=\mu_{z}=1.5, \eta_{x}=0.53, \eta_{z}=0.39$ ).

(a)

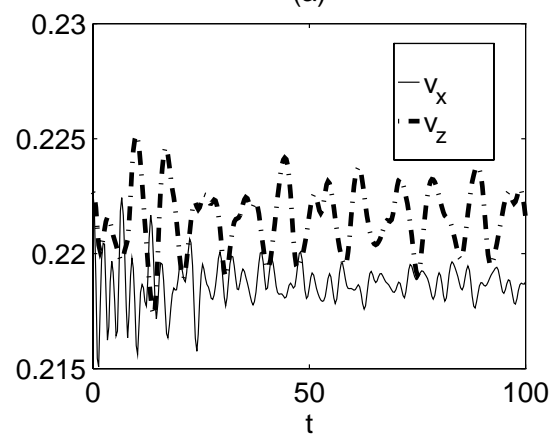

being introduced, similar to the observations in [16]. The field energy is predominantly transferred into the plane where the thermal energy is initially smaller. The observed anisotropy is a function of the initial rms mismatch and the space charge. It slightly increases with increasing mismatch and/or space charge. However, even for high mismatches, the introduced anisotropy is relatively small, as shown in Fig. 21 for initial rms mismatch $\mu_{x}=\mu_{y}=\mu_{z}=1.5$. Since the redistribution process in beams with nonstationary distributions is equivalent to an initial mismatch, it also leads to an anisotropy between the final states. The largest anisotropy is once again observed for a Gaussian distribution, as can be seen in Fig. 22. Figure 23 shows the introduced anisotropy for the 6D uniform and 6D Gaussian distribution with the initial mismatch $\mu_{x}=\mu_{y}=\mu_{z}=1.5$.

\section{SUMMARY}

Most of the previous studies were concerned with halos in long beams. In the current work we address the question of halo formation in a beam bunch which is of particular interest for the Accelerator Production of Tritium project where relatively short bunches are proposed [18].

We recently constructed, analytically and numerically, a new class of $6 \mathrm{D}$ phase space stationary distributions for an azimuthally symmetric beam bunch of arbitrary charge in the shape of a prolate spheroid. The stationary distribution allowed us to study the halo development mechanism in $3 \mathrm{D}$ beam bunches where no phase space redistribution occurs.

After we established the parameters which lead to halo formation in 3D beam bunches for the self-consistent $6 \mathrm{D}$ phase space stationary distribution [12], in this paper we explored rms matched distributions which are not selfconsistent to determine the extent to which the relatively rapid redistribution of the $6 \mathrm{D}$ phase space contributes to the formation of halos. The redistribution process and its influence on halo formation is described in detail in Sec. III. We also found that the effect of coupling

(b)

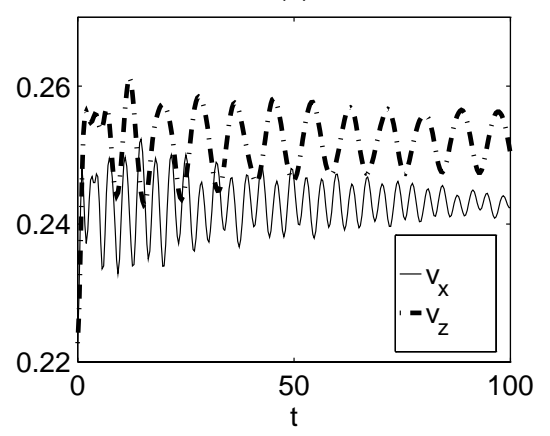

FIG. 22. rms velocities (in arbitrary units) for initially matched nonstationary distributions $\left(c / a=3, \mu_{x}=\mu_{y}=\mu_{z}=1.0, \eta_{x}=\right.$ 0.53, $\eta_{z}=0.39$ ): (a) 6D uniform distribution and (b) 6D Gaussian distribution 
(a)

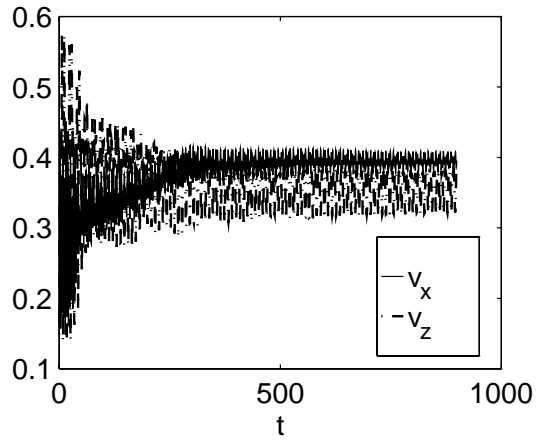

(b)

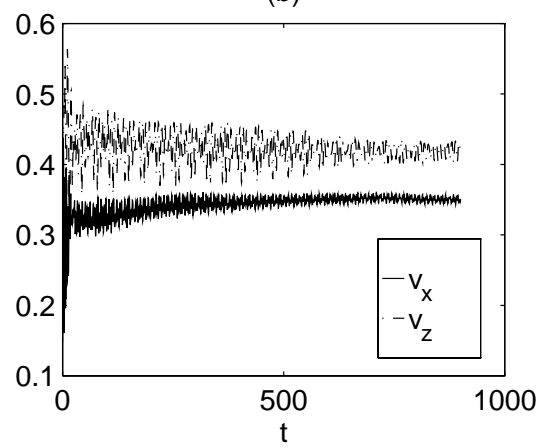

FIG. 23. rms velocities (in arbitrary units) for initially mismatched nonstationary distributions $\left(c / a=3, \mu_{x}=\mu_{y}=\mu_{z}=\right.$ $1.5, \eta_{x}=0.53, \eta_{z}=0.39$ ): (a) 6D uniform distribution and (b) 6D Gaussian distribution

between the $r$ and $z$ planes is very important in the halo development mechanism and can lead to serious consequences, especially for a very short beam bunch.

\section{ACKNOWLEDGMENTS}

We acknowledge the support of the U.S. Department of Energy, Division of High Energy Physics, and Division of Mathematical, Information, and Computational Sciences. For this research we used resources of the National Energy Research Scientific Computing Center, which is supported by the DOE Office of Energy Research. We thank Tom Wangler for helpful conversations. In addition, R. L. G. and A. V. F. thank Andy Jason and the LANSCE1 group for their hospitality during part of these studies.

[1] M. Reiser, in Proceedings of the 1991 Particle Accelerator Conference, San Francisco, edited by L. Lizama et al. (IEEE, Piscataway, NJ, 1991), p. 2497; A. Cucchetti, M. Reiser, and T. Wangler, ibid., p. 251; J.S. O'Connell, T.P. Wangler, R. S. Mills, and K. R. Crandall, in Proceedings of the 1993 Particle Accelerator Conference, Washington, DC, edited by S.T. Corneliussen (IEEE, Piscataway, NJ, 1993), p. 3657.

[2] R. A. Jameson, Los Alamos Report No. LA-UR-93-1209 (unpublished).

[3] R. A. Jameson, in Proceedings of the Joint US-CERNJapan International School, Maui, Hawaii, 1994 (World Scientific, Singapore, 1996), pp. 530-560.
[4] R. L. Gluckstern, Phys. Rev. Lett. 73, 1247 (1994).

[5] A. Riabko, M. Ellison, X. Kang, S. Y. Lee, D. Li, J. Y. Liu, X. Pei, and L. Wang, Phys. Rev. E 51, 3529 (1995).

[6] M. Reiser, Theory and Design of Charged Particle Beams (Wiley, New York, 1994).

[7] R. L. Gluckstern, W-H. Cheng, S. S. Kurennoy, and H. Ye, Phys. Rev. E 54, 6788 (1996).

[8] R. L. Gluckstern, W-H. Cheng, and H. Ye, Phys. Rev. Lett. 75, 2835 (1995).

[9] R.L. Gluckstern and S.S. Kurennoy, in Proceedings of the 1997 Particle Accelerator Conference, Vancouver, Canada (IEEE, Piscataway, NJ, 1998), p. 1950.

[10] J. J. Barnard and S. M. Lund, in Proceedings of the 1997 Particle Accelerator Conference, Vancouver, Canada (IEEE, Piscataway, NJ, 1998), p. 1929; S. M. Lund and J. J. Barnard, ibid., p. 1932.

[11] H. Okamoto and M. Ikegami, Phys. Rev. E 55, 4694 (1997).

[12] R. L. Gluckstern, A. V. Fedotov, S. S. Kurennoy, and R. D. Ryne, Phys. Rev. E 58, 4977 (1998).

[13] E. Forest, J. Bengtsson, and M. F. Reusch, Phys. Lett. A 158, 99 (1991).

[14] R. W. Hockney and J. W. Eastwood, Computer Simulation Using Particles (Adam Hilger, New York, 1988).

[15] R. Ferrell and E. Bertschinger, Int. J. Mod. Phys. C 5, 933 (1994).

[16] I. Hofmann and J. Struckmeier, Part. Accel. 21, 69-98 (1987).

[17] T.P. Wangler, K. R. Crandall, E. R. Gray, and S. Nath, in Proceedings of the 1997 Particle Accelerator Conference, Vancouver, Canada (IEEE, Piscataway, NJ, 1998), p. 915.

[18] APT Conceptual Design Report, Los Alamos Report No. LA-UR-97-1329, 1997. 